Pesq. Vet. Bras. 29(5):404-408, maio 2009

\title{
Intoxicação natural e experimental por Nerium oleander (Apocynaceae) em bovinos no Rio Grande do Sul ${ }^{1}$
}

\author{
Pedro M.O. Pedroso², Paulo M. Bandarra², Pedro S. Bezerra Júnior ${ }^{3}$, Djeison \\ L. Raymundo², Mauro R. Borba ${ }^{2}$, Juliano S. Leal ${ }^{2}$ e David Driemeier ${ }^{2}$
}

\begin{abstract}
Pedroso P.M.O., Bandarra P.M., Bezerra Júnior P.S., Raymundo D.L., Borba M.R., Leal J.S. \& Driemeier D. 2009. [Natural and experimental poisoning by Nerium oleander (Apocynaceae) in cattle in Rio Grande do Sul.] Intoxicação natural e experimental por Nerium oleander (Apocynaceae) em bovinos no Rio Grande do Sul. Pesquisa Veterinária Brasileira 29(5):404-408. Setor de Patologia Veterinária, Faculdade de Veterinária, Universidade Federal do Rio Grande do Sul, Av. Bento Gonçalves 9090, Porto Alegre, RS 91540-000, Brazil. E-mail: davetpat@ufrgs.br

This paper describes natural and experimental poisoning of cattle by Nerium oleander in Rio Grande do Sul. Two out of eight cattle died acutely after consumption of leaves of Nerium oleander, branches of which had been cut and placed into a paddock where the animals were kept. An affected cow did not show clinical signs, but a 4-month-old calf presented lateral recumbence, paddling, vocalization and death. Main gross findings in the cow naturally poisoned and in two experimentally intoxicated heifers were observed in the heart and included hemorrhages in the left atrium, clots and hemorrhages in the left ventricular endocardium, and pale areas in the interventricular septum and ventricular myocardium. Histologically, there was coagulation necrosis of individual cardiac fibers or small groups of fibers, characterized by enhanced cytoplasmic eosinophily and picnotic nuclei. These lesions were most severe in the papillary muscle. The diagnosis was based on presence of the trimmed $N$. oleander in the paddock where the animals stayed, evidence of consumption of the plant, consistent clinical and pathological findings, and experimental reproduction of the disease through oral administration of 0.5 and $1.0 \mathrm{~g} / \mathrm{kg}$ of its green leaves to two cattle.
\end{abstract}

INDEX TERMS: Nerium oleander, ornamental plant poisoning, cattle, heart lesions.

RESUMO.- Descreve-se a intoxicação natural e experimental por Nerium oleander em bovinos no Rio Grande do Sul, Brasil. Dois de oito bovinos morreram subitamente após consumirem folhas de galhos de $N$. oleander que haviam sido podados e jogados no piquete onde os animais eram mantidos. Não foram observados sinais clínicos em uma vaca, mas um terneiro de 4 meses de idade apresentou decúbito lateral, movimentos de pedalagem, vocalização e morte. As alterações macroscópicas mais

\footnotetext{
${ }^{1}$ Recebido em 27 de novembro de 2008.

Aceito para publicação em 29 de dezembro de 2008.

2 Setor de Patologia Veterinária (SPV), Departamento de Patologia Clínica Veterinária, Faculdade de Veterinária, Universidade Federal do Rio Grande do Sul (UFRGS), Av. Bento Gonçalves 9090, Porto Alegre, RS 95320-000, Brasil. "Autor para correspondência: davetpat@ufrgs.br

${ }^{3}$ SPV, Departamento de Patologia Veterinária, Universidade Federal de Lavras (UFLA), Cx. Postal 3037, Lavras, MG, 37200-000, Brasil.
}

importantes observadas na vaca morta naturalmente e em duas novilhas intoxicadas experimentalmente ocorreram no coração que apresentava petéquias e equimoses no átrio esquerdo, coágulos e hemorragias no endocárdio do ventrículo esquerdo e áreas levemente pálidas no septo interventricular e em porções do miocárdio dos ventrículos. $\mathrm{Na}$ histologia, havia necrose de coagulação de fibras cardíacas individuais e de pequenos agrupamentos, caracterizada por aumento de eosinofilia citoplasmática e núcleos picnóticos. Essas lesões eram mais acentuadas no músculo papilar. O diagnóstico foi fundamentado na presença da planta no potreiro onde estavam os animais, quadro clínico-patológico compatível e reprodução experimental em dois bovinos nas doses de 0,5 e $1,0 \mathrm{~g} / \mathrm{kg}$ de folhas frescas da planta.

TERMOS DE INDEXAÇÃO: Nerium oleander, intoxicação por planta ornamental, bovinos, lesões cardíacas. 


\section{INTRODUÇÃO}

Nerium oleander, popularmente conhecida como "espirradeira" (Armién et al. 1994) e originária do Mediterrâneo e Ásia (Oryan et al. 1996, Kellerman et al. 2005), é cultivada como planta ornamental em muitas partes do Mundo (Aslani et al. 2004). Todas as partes da planta contêm glicosídeos cardiotóxicos (Armién et al. 1994, Osweiler 1995), oleandrina e neriina (Galey 2004). A intoxicação geralmente ocorre quando a planta ou seus galhos são cortados e deixados ao alcance dos animais (Riet-Correa \& Méndez 2007). Intoxicações espontâneas foram registradas em bovinos (Thimmiah 1972, Assis et al. 2008), equinos (Hughes et al. 2002), ovinos e caprinos (Kellerman et al. 2005, Barbosa et al. 2008). A intoxicação em humanos tem sido descrita em decorrência de ingestão acidental ou tentativa de suicídio (Pietsch et al. 2005). Sinais clínicos são observados entre 1 e 24 horas após a ingestão (Baskin et al. 2007) e incluem arritmias (Tokarnia et al. 1996), vômito, diarréia (às vezes sanguinolenta), ataxia, extremidades frias, dispnéia, paralisia, coma e morte (Baskin et al. 2007). Este estudo relata a intoxicação natural por Nerium oleander em bovinos no Rio Grande do Sul e descreve aspectos da reprodução experimental da doença com especial referência à caracterização do quadro clínico-patológico e mapeamento das principais áreas cardíacas afetadas.

\section{MATERIAL E MÉTODOS}

Dados epidemiológicos foram obtidos com o proprietário e o veterinário de campo, durante visita à propriedade onde ocorreram as intoxicações. Devido às evidências da intoxicação por Nerium oleander, decidiu-se reproduzir experimentalmente a doença em bovinos de cerca 1 ano de idade, da raça Hereford. O experimento foi realizado nas dependências do Setor de Patologia Veterinária da Universidade Federal do Rio Grande do Sul (SPV-UFRGS), Porto Alegre, RS. Os animais foram mantidos individualmente em um curral com feno de alfafa e água ad libitum. As folhas de $N$. oleander foram coletadas na propriedade onde ocorreram as mortes e administradas via oral, em doses de $1,0 \mathrm{~g} / \mathrm{kg}$ e $0,5 \mathrm{~g} / \mathrm{kg}$ de peso corporal, respectivamente, para duas novilhas (Bovinos 2 e 3), conforme procedimentos relatados anteriormente (Tokarnia et al. 1996, RietCorrea \& Méndez 2007). Exames clínicos foram realizados periodicamente. Durante as necropsias, fragmentos de diversos órgãos dos três bovinos (um da intoxicação natural e dois da reprodução experimental) foram coletados e fixados em formol tamponado a $10 \%$, processados rotineiramente para exame histológico e corados pela hematoxilina e eosina (HE). Cortes seriados foram feitos nos ventrículos direito e esquerdo, átrios direito e esquerdo, músculo papilar e septo interventricular. Atribuíram-se os graus: ausente (-), leve (+), moderado (++) e acentuado $(+++)$ às lesões. Adicionalmente, amostras de sangue dos casos experimentais foram coletadas antes e após a administração de Nerium oleander, dessoradas e encaminhadas para a dosagem de creatina quinase do músculo cardíaco (CK-MB) (Diniz et al. 2007).

\section{Casos naturais}

\section{RESULTADOS}

As mortes ocorreram no mês de agosto de 2007, em uma propriedade rural localizada no município de Viamão,
Rio Grande do Sul. O lote de bovinos composto por um touro, três vacas e quatro bezerros era mantido em potreiro de campo nativo com baixa disponibilidade de forragem. O lote recebia uma suplementação total de $50 \mathrm{~kg} / \mathrm{dia}$ de uma mistura de casquinha de soja, farelo de trigo e sal mineral.

Um bezerro mestiço de 4 meses de idade morreu subitamente após consumir folhas dos galhos podados de Nerium oleandere apresentou decúbito lateral, movimentos de pedalagem e vocalização. Esse bezerro não foi necropsiado porque havia morrido dois dias antes da visita. Um bovino fêmea com 3 anos de idade, da raça Brahman (Bovino 1) foi encontrada morta ao amanhecer, sem observação prévia de sinais clínicos. Essa vaca foi descrita como dominante e a mais voraz do lote. Havia congestão de mucosas (ocular, bucal, vulvar). Alterações macroscópicas observadas no coração incluíram petéquias e equimoses no átrio esquerdo, coágulos e hemorragias no endocárdio do ventrículo esquerdo, áreas levemente pálidas no septo interventricular e em algumas porções do miocárdio dos ventrículos. Observou-se também espuma na porção final da traquéia e nos brônquios, fígado vermelho-escuro, rins pálidos, avermeIhamento da mucosa do retículo e rúmen, onde havia considerável quantidade de folhas de $N$. oleandere seus fragmentos. Durante a visita à propriedade, constatou-se que um arbusto de $N$. oleander havia sido podado e seus gaIhos jogados no potreiro onde os animais eram mantidos. Esses galhos podados apresentavam sinais de haverem sido consumidos. Fezes frescas de bovino foram encontradas nas proximidades dos galhos (Fig.1).

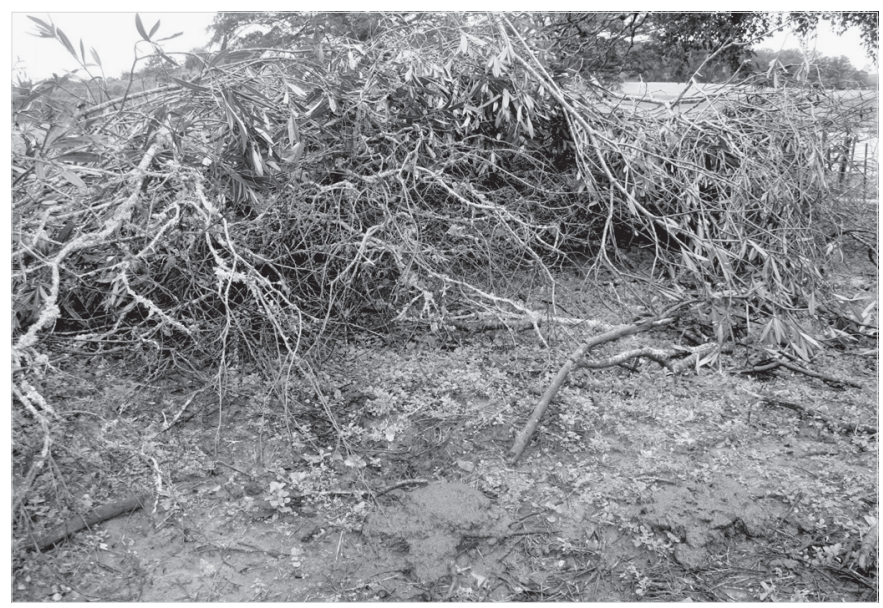

Fig.1. Intoxicação natural por Nerium oleander. Local onde foram colocados os galhos podados. Galhos da planta com sinais de haverem sido consumidos e fezes no entorno, demonstrando o acesso dos bovinos.

Quadro 1. Evolução do quadro clínico e desfecho. Intoxicação experimental por Nerium oleander em bovinos

\begin{tabular}{|c|c|c|c|c|c|c|}
\hline $\begin{array}{c}\text { Bovino } \\
\mathrm{n}^{\circ}\end{array}$ & $\begin{array}{l}\text { Idade } \\
\text { (anos) }\end{array}$ & $\begin{array}{r}\text { Peso } \\
\text { (kg) }\end{array}$ & $\begin{array}{l}\text { Dose } \\
\text { (g/kg) }\end{array}$ & $\begin{array}{c}\text { Início dos } \\
\text { sinais clínicos }\end{array}$ & Evolução & Desfecho \\
\hline 2 & 1,5 & 180 & 1,0 & $29 \mathrm{~min}$ & $62 \mathrm{~min}$ & Morreu \\
\hline 3 & 1 & 245 & 0,5 & $18 \mathrm{~h} 15 \mathrm{~min}$ & $25 \mathrm{~h} 50 \mathrm{~min}$ & Morreu \\
\hline
\end{tabular}




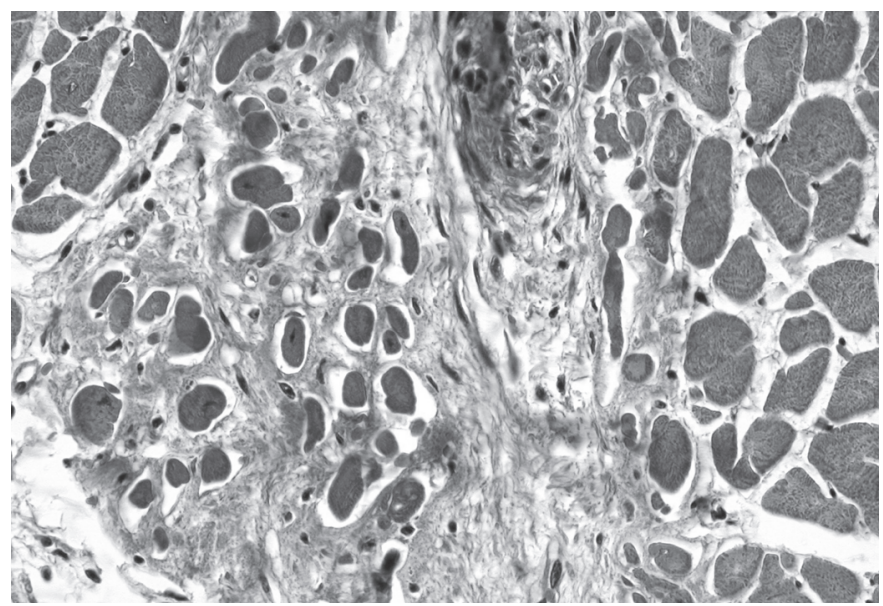

Fig.2. Intoxicação natural por Nerium oleander (bovino 1). Coração. Músculo papilar com necrose de coagulação de fibras individuais e de pequenos agrupamentos caracterizada por aumento de eosinofilia citoplasmática e núcleos picnóticos. HE, obj.40x.

Quadro 2. Distribuição das principais lesões microscópicas observadas no coração de bovinos intoxicados por Nerium oleander

\begin{tabular}{|c|c|c|c|c|}
\hline $\begin{array}{l}\text { Bovino } \\
\text { no. }\end{array}$ & $\begin{array}{c}\text { Secções } \\
\text { histológicas }^{a}\end{array}$ & $\begin{array}{c}\text { Aumento da } \\
\text { eosinofilia } \\
\text { de fibras }\end{array}$ & Picnose & $\begin{array}{c}\text { Infiltrado } \\
\text { mononuclear }\end{array}$ \\
\hline \multirow[t]{6}{*}{1} & VE & + & + & + \\
\hline & VD & + & + & + \\
\hline & $A E$ & NA & NA & NA \\
\hline & AD & + & + & ++ \\
\hline & MP & ++ & ++ & - \\
\hline & SI & + & + & +++ \\
\hline \multirow[t]{6}{*}{2} & VE & ++ & ++ & + \\
\hline & VD & + & + & - \\
\hline & $A E$ & + & + & - \\
\hline & AD & + & + & - \\
\hline & MP & ++ & ++ & - \\
\hline & SI & - & - & + \\
\hline \multirow[t]{6}{*}{3} & VE & + & + & - \\
\hline & VD & - & - & + \\
\hline & $A E$ & - & - & - \\
\hline & AD & - & - & - \\
\hline & MP & +++ & +++ & + \\
\hline & SI & - & - & - \\
\hline
\end{tabular}

a $\mathrm{VE}=$ ventrículo esquerdo, $\mathrm{VD}=$ ventrículo direito, $\mathrm{AE}$ = átrio esquerdo, $\mathrm{AD}=$ átrio direito, $\mathrm{MP}=$ músculo papilar, $\mathrm{SI}=$ Septo interventricular;, + lesão leve, ++ lesão moderada, +++ lesão acentuada, - sem lesão, NA = não avaliado.

Ao exame microscópico, observou-se necrose de coagulação de fibras cardíacas individuais em pequenos agrupamentos, caracterizada por aumento de eosinofilia citoplasmática e núcleos picnóticos (Fig.2). Essas lesões eram mais acentuadas no músculo papilar (Quadro 2). Algumas áreas apresentavam material proteináceo no interstício e hemorragia subendocárdica. O rúmen apresentava degeneração hidrópica das camadas superficiais do epitélio. No fígado, havia congestão difusa acentuada e, no baço, infiltrado linfohistiocitário nos folículos e hemossiderina no interior de macrófagos.

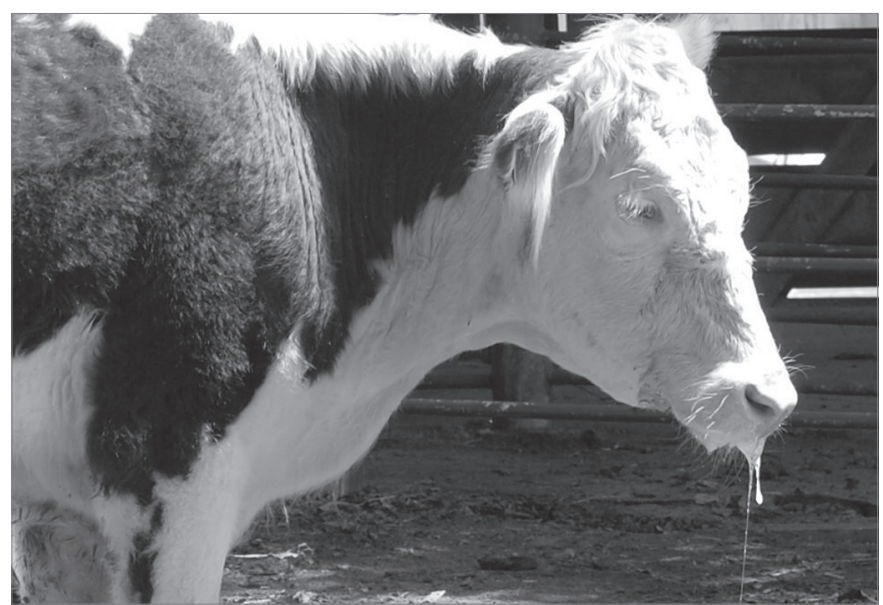

Fig.3. Intoxicação experimental por Nerium oleander. Bovino 2 apresentando sialorréia.

\section{Reprodução experimental}

O delineamento experimental, a evolução do quadro clínico nos animais intoxicados e o desfecho do experimento com $N$. oleander em bovinos são mostrados no Quadro 1. Antes da dosagem da planta, as frequências respiratória e cardíaca foram de 28 e 60 movimentos por minuto, respectivamente. O Bovino 2 teve uma evolução clínica de 62 minutos e morreu 91 minutos após a administração da planta. Vinte e nove minutos após a ingestão, o animal apresentou sialorréia (Fig.3), seguindo com micção frequente, ranger de dentes e tremores musculares generalizados. Houve aumento da frequência respiratória para 148, dificuldade de locomoção, regurgitação de conteúdo ruminal, queda brusca do animal ao chão, decúbito lateral, movimentos de pedalagem, intensa taquicardia, mugidos e morte. O Bovino 3 teve evolução clínica de 25 horas e 50 minutos; morreu 44 horas e cinco minutos após a administração da planta. Aumento das frequências cardíaca e respiratória foram os primeiros sinais clínicos observados e ocorreram 18 horas e $15 \mathrm{mi}-$ nutos após a administração da planta. Trinta minutos antes da morte, o animal apresentou relutância em caminhar, queda, decúbito lateral, movimentos de pedalagem, tremores musculares generalizados, mugidos e morte.

Os principais achados macroscópicos foram observados no coração e incluíram petéquias e equimoses no epicárdio, coágulos e hemorragias no endocárdio do ventrículo esquerdo. Espuma na porção final da traquéia e brônquios, fígado de coloração vermelho-escura, rins pálidos e avermelhamento da mucosa do abomaso, rúmen e retículo foram outros achados de necropsia.

No Bovino 2, as principais alterações histopatológicas foram aumento da eosinofília, picnose e infiltrado mononuclear multifocal leve em fibras cardíacas individuais ou em pequenos grupos. Observaram-se também parasitas Sarcocystis sp. nas fibras cardíacas, fígado com congestão difusa acentuada e abomaso com congestão difusa leve. No Bovino 3, o coração apresentou tumefação, vacuolização, extensas áreas de necrose hialina, aumento 


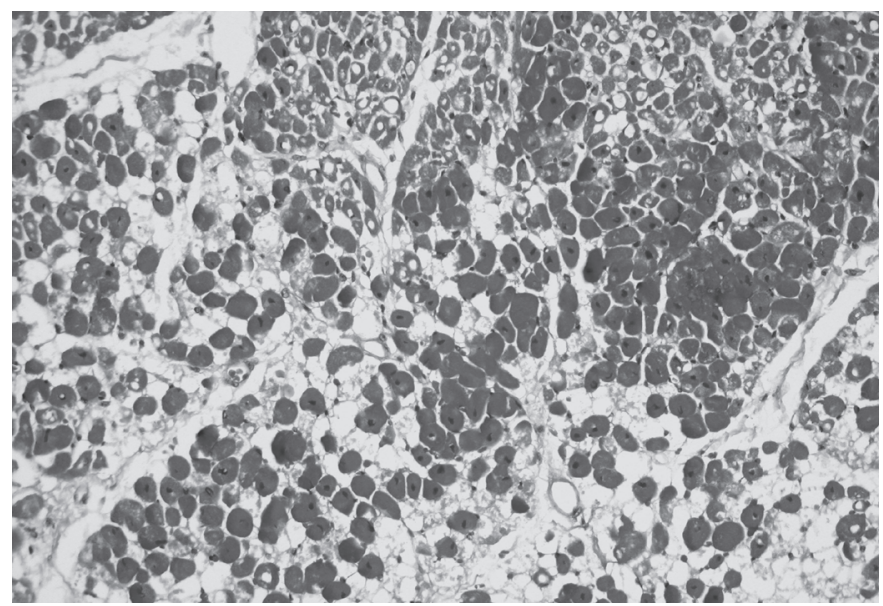

Fig.4. Intoxicação experimental por Nerium oleander (bovino 3). Coração. Músculo papilar com extensas áreas de necrose hialina, aumento da eosinofilia, picnose e vacúolos citoplasmáticos perinuclear. HE, obj.20x.

Quadro 3. Resultados de CK-MB. Intoxicação experimental por Nerium oleander em bovinos

\begin{tabular}{ccc}
\hline Bovino no. & \multicolumn{2}{c}{ CK-MB (U/I) } \\
\cline { 2 - 3 } & Antes & Depois \\
\hline 2 & 158 & 402 \\
3 & 226 & 285
\end{tabular}

da eosinofilia, picnose e vacúolos citoplasmáticos perinucleares (Fig.4). Entre as miofibras, observou-se material eosinofílico frouxo. As lesões eram mais prevalentes nas proximidades do endocárdio. No septo interventricular, observaram-se hemorragia subendocárdica, vacuolização citoplasmática e perinuclear nas fibras próximas ao endocárdio. Omaso, rúmen e retículo apresentaram degeneração hidrópica na camada superficial, por vezes acompanhada de infiltrado neutrofílico e intestino com congestão moderada. O grau de severidade das lesões microscópicas cardíacas está apresentado no Quadro 2. Houve aumento significativo de CK-MB do bovino 2. Os resultados estão demonstrados no Quadro 3.

\section{DISCUSSÃO}

Casos de intoxicação por plantas ornamentais em animais de fazenda são raros (Tokarnia et al. 2000) provavelmente devido à baixa palatabilidade e às restrições ao acesso dos animais a essas plantas (Tokarnia et al. 2000, Soto-Blanco et al. 2006). Entretanto, a ingestão de pequenas quantidades de folhas é suficiente para causar a morte de animais (Tokarnia et al. 1996). Os relatos de casos de intoxicação natural por Nerium oleander em bovinos são escassos (Soto-Blanco et al. 2006, Assis et al. 2008, Barbosa et al. 2008) e os fatores epidemiológicos frequentemente incluem pouca disponibilidade de alimento e ingestão acidental da planta (Thimmiah 1972, Assis et al. 2008). A dominância e a voracidade da vaca e a curiosidade do bezerro, mortos naturalmente, podem ter contribuído para a intoxicação.
Tanto 0,5 quanto $1,0 \mathrm{~g} / \mathrm{kg}$ de folhas de $N$. oleander produziram sinais clínicos e morte dos animais experimentais. O quadro clínico-patológico observado na intoxicação por $N$. oleander é causado pela ação de glicosídeos cardíacos que atuam sobre a bomba de sódio e potássio, resultando em diminuição do $\mathrm{K}$ intracelular e aumento do $\mathrm{K}$ sérico, o que causa diminuição da condutividade elétrica no coração, arritmias e eventual parada cardíaca (RietCorrea \& Méndez 2007). As lesões microscópicas cardíacas foram semelhantes às descritas na intoxicação experimental em bovinos (Oryan et al. 1996, Tokarnia et al. 1996) e ovinos (Armién et al. 1994). Tanto nos casos naturais, como nos experimentais, as lesões foram mais significativas no músculo papilar do coração. As dosagens de CKMB dos bovinos experimentais foram aumentadas, principalmente do bovino 2 que recebeu maior dose da planta $(1,0 \mathrm{~g} / \mathrm{kg})$. Nos episódios de necrose do miocárdio, há liberação da isoenzima CK-MB para o meio extracelular, o que tem valor diagnóstico (Diniz et al. 2007).

No Brasil, é frequente a ocorrência de "morte súbita" em bovinos por ingestão de plantas tóxicas. Essas mortes repentinas em geral se manifestam sem quadro clínico prévio e se caracterizam pela ausência de achados macroscópicos na necropsia (Gava et al. 1998, Tokarnia et al. 2000). É importante considerar o diagnóstico diferencial por plantas ornamentais como Thevetia peruviana e Rhododendron spp. (Tokarnia et al. 1996) que cursam com sinais clínicos semelhantes aos observados, além de plantas que causam morte súbita como Palicourea marcgravii, $P$. aeneofusca, $P$. juruana, $P$. grandiflora, Arrabidaea bilabiata, A. japurensis, Pseudocalymma elegans, Mascagnia rigida, M. elegans, M. pubiflora e $M$. aff. rigida (Tokarnia et al. 2000, Riet-Correa \& Méndez 2007). Na região Sul, é importante realizar o diagnóstico diferencial de Mascagnia spp., que é encontrada no Litoral de Santa Catarina (Gava et al. 1998) e Rio Grande do Sul (Riet-Correa \& Méndez 2007, Driemeier, comunicação pessoal). No entanto, nos casos de intoxicação por essas plantas, não são observadas alterações macroscópicas significativas e, na histologia, observa-se, em alguns animais, degeneração hidrópica no epitélio tubular renal (Tokarnia et al. 1990). Em casos de suspeita de intoxicação por $N$. oleander é importante realizar cortes seriados do coração para identificar microscopicamente as áreas onde ocorrem mais lesões e definir o diagnóstico através dos fatores epidemiológicos.

Embora a intoxicação por $N$. oleander em animais de interesse pecuário não seja comum, a ampla distribuição dessa planta ornamental no Brasil tem predisposto o surgimento de casos de intoxicação. O consumo de $N$. oleander deve ser considerado em casos de mortes súbitas em bovinos no Brasil.

Agradecimentos.- Ao Conselho Nacional de Desenvolvimento Científico e Tecnológico (CNPq) e à Coordenação de Aperfeiçoamento de Pessoal de Nível Superior (CAPES) pelo apoio financeiro. Os autores agradecem ao professor Cláudio Estêvão Farias da Cruz (UFRGS) pela revisão deste artigo. 


\section{REFERÊNCIAS}

Armién A.G., Peixoto P.V., Barbosa J.D. \& Tokarnia C.H. 1994. Intoxicação experimental por Nerium oleander (Apocinaceae) em ovinos. Pesq. Vet. Bras. 14:85-93.

Aslani M.R., Movassaghi A.R., Mohri M., Abbasian A. \& Zarehpour M. 2004. Clinical and pathological aspects of experimental oleander (Nerium oleander) toxicosis in sheep. Vet. Res. Commun. 28:609-616.

Assis T.S., Galiza G.J.N., Neto T.S.O., Medeiros R.M.T., Dantas A.F. \& Riet-Correa F. 2008. Intoxicação por Nerium oleander em bovino no Semi-Árido Paraibano. Anais Encontro Nacional de Diagnóstico Veterinário, Campo Grande, MS, p.209-210.

Barbosa R.R., Fontenele-Neto J.D. \& Soto-Blanco B. 2008. Toxicity in goats caused by (Nerium oleander). Res. Vet. Sci. 85:279-281.

Baskin S.I., Czerwinski S.E., Anderson J.B. \& Sebastian M.M. 2007. Cardiovascular toxicity, p.193-205. In: Gupta R.C. (Ed.), Veterinary Toxicology. Elsevier, New York, NY.

Diniz P.P.V.P., Schwartz D.S. \& Collicchio-Zuanaze R.C. 2007. Cardiac trauma confirmed by cardiac markers in dogs: Two cases reports. Arq. Bras. Med. Vet. Zootec. 59:85-89.

Galey F.D. 2004. Cardiac glycosides, p.386-388. In: Plumlee K.H. (Ed.), Clinical Veterinary Toxicology. Mosby, St Louis, Missouri.

Gava A., Cristani J., Branco J.V., Neves D.S., Mondadori A.J. \& Sousa R.S. 1998. Mortes súbitas em bovinos causadas pela ingestão de Mascagnia sp (Malpighiaceae), no Estado de Santa Catarina. Pesq. Vet. Bras. 18:16-20.

Hughes K.J., Dart A.J. \& Hodgson D.R. 2002. Suspected Nerium oleander (Oleander) poisoning in a horse. Aust. Vet. J. 80:412-415.
Kellerman T.S., Coetzer J.A.W., Naudé T.W. \& Botha C.J. 2005. Plant Poisonings and Mycotoxicoses. Oxford University Press, Cape Town, RSA. 310p.

Oryan A., Maham M., Rezakhani A. \& Maleki M. 1996. Morphological studies on experimental oleander poisoning in cattle. J. Vet. Med. A 43:625-634.

Osweiler G.D. 1995. Toxicology. Lippincott Williams and Wilkins, Philadelphia. 491p.

Pietsch J., Oertel R., Trautmann S., Schulz K., Kopp B. \& Dreâler. 2005. A non-fatal oleander poisoning. Int. J. Legal Med. 119:236-240.

Riet-Correa F. \& Méndez M.C. 2007. Intoxicações por plantas e micotoxinas, p.99-219. In: Riet-Correa F., Schild A.L., Lemos R.A.A. \& Borges J.R.J. (Eds), Doenças de Ruminantes e Eqüídeos. Vol.2. Editora Pallotti, Santa Maria, RS.

Soto-Blanco B., Fontenele-Neto J.D., Silva D.M., Reis P.F.C.C. \& Nóbrega J.E. 2006. Acute cattle intoxication from Nerium oleander pods. Trop. Anim. Hlth Prod. 38:451-454.

Thimmiah K. 1972. Nerium poisoning in cattle. Indian Vet. J. 49:942946.

Tokarnia C.H., Peixoto P.V. \& Döbereiner J. 1990. Poisonous plants affecting heart function of cattle in Brazil. Pesq. Vet. Bras. 10:1-10.

Tokarnia C.H., Armién A.G., Peixoto P.V., Barbosa J.D., Brito M.F. \& Döbereiner J. 1996. Estudo experimental sobre a toxidez de algumas plantas ornamentais em bovinos. Pesq.Vet. Bras. 16:5-20.

Tokarnia C.H., Döbereiner J. \& Peixoto P.V. 2000. Plantas Tóxicas do Brasil. Editora Helianthus, Rio de Janeiro. 310p. 